\title{
Neutron energy analysis by silicon prisms
}

\author{
J. Schulz ${ }^{1 \mathrm{a}}$, F. Ott ${ }^{\mathrm{b}}$, Ch. Hülsen ${ }^{\mathrm{a}}$, Th. Krist ${ }^{\mathrm{a}}$ \\ ${ }^{a}$ Helmholtz-Zentrum Berlin für Materialien und Energie, Hahn-Meitner-Platz 1, 14109 \\ Berlin, Germany \\ ${ }^{b}$ Laboratoire Leon Brillouin, Bât 563 CEA Saclay, 91191 Gif sur Yvette Cedex, France
}

\begin{abstract}
Neutron energy analysing techniques allow to measure at different wavelengths at the same time thus avoiding losses due to monochromatization. We built and tested a refractive energy analysing device made from small prisms, where losses only occur due to the attenuation in the material.

We measured the refraction and the transmission of $\mathrm{MgF}_{2}$ and $\mathrm{Si}$ prisms at the V14 reflectometer in Berlin at $4.9 \AA$ to check their applicability. The experimentally determined linear attenuation coefficients are $0.055 \mathrm{~cm}^{-1}$ for the $\mathrm{MgF}_{2}$ and $0.03 \mathrm{~cm}^{-1}$ for the Si prisms. An energy analyser consisting of silicon prism layers was measured at the EROS reflectometer at the LLB in a white neutron beam. The useful wavelength band was 2.4 to $7.6 \AA$. At $6.7 \AA$ a wavelength resolution of $5 \%$ and a transmission of $53 \%$ were achieved. The surface roughness of the prisms could be determined to be $(0.011 \pm 0.006) \mathrm{deg}$.
\end{abstract}

Keywords: Neutron Optics, Energy analysis, Prisms

\section{Introduction}

Neutron reflectometry is an important technique to measure structure and composition close to the surface of a sample. There are principally two options to measure the ratio of the incoming and the reflected beam as a function of the wavevector transfer. In reflectometers working in time-offlight mode a chopper is used to encode the neutron wavelengths in time and the incident angle of the neutron beam is fixed. The measurement of the

\footnotetext{
${ }^{1}$ Corresponding author. Tel.: +49 30806243048.

E-mail address: jennifer.schulz@helmholtz-berlin.de (J. Schulz)
} 
reflection is done for different wavelengths. In reflectometers working in the monochromatic mode a crystal is used to select a fixed wavelength and the measurement is performed at different angles.

Since the use of monochromators and choppers causes high losses in the neutron flux, several optical elements had been investigated to substitute these elements [1]. One option is to use a white beam and split the neutron wavelengths in space. This can be accomplished by a magnetic field gradient [2] or the refraction of the neutron beam in matter can be used [3][4], e.g. the refraction by a row of prisms, as pioneered in the field of x-rays by $\mathrm{W}$. Jark [5].

\section{Theory}

Here we want to derive an expression for the wavelength resolution and identify the effects contributing to the angular broadening of the neutron beam.

For prism rows the refraction angle and thus the resolution increases with the number of prisms. The angular dispersion of the system is the change of the refracted angle according to the change in the index of refraction $n$, which is

$$
n=1-\frac{\lambda^{2}}{2 \pi} N b,
$$

where $\lambda$ is the wavelength of the neutron and $\mathrm{Nb}$ the scattering length density of the prism material. The refraction angle $\varphi$ is given by Snells law

$$
\varphi=\sin ^{-1}(n \sin \alpha)
$$

where $\alpha$ is the incident angle. Since the angle increases in very good approximation linearly with the number of prism surfaces, the deflection of the whole prism row is increased by a factor of 2 times the number of prisms $i$. Thus the angular dispersion is

$$
\frac{d \varphi_{i}}{d \lambda} \approx \frac{d\left(2 i \sin ^{-1}(n \sin (\alpha))\right.}{d \lambda}=2 i \frac{d \varphi_{i=1}}{d \lambda}
$$

and the wavelength resolution of a prism system is given by

$$
\frac{d \lambda}{\lambda} \approx \frac{d \varphi}{2 i} \frac{\left(1-n^{2}(\sin \alpha)^{2}\right)^{1,5}}{(\sin \alpha)^{3} n \frac{\lambda^{2} N b}{\pi}} .
$$


The variable $\varphi$ covers all effects that broaden the angular distribution of the neutron beam. These effects are the incoming beam divergence $\Theta_{\text {Div }}$ and the scattering by surface roughness $\Theta_{\text {Rough }}$. Another effect is the total reflection $\Theta_{\text {Refl }}$, which occurs when the neutrons leave the prism layer they entered and hit the flat back side of the next prism layer at an angle smaller than the critical angle. If this angle is larger than the critical angle small angle refraction occurs and broadens the beam by $\Theta_{\text {Refr }}$. At last the resolution of the detector $\Theta_{D e t}$ also increases the effective broadening. Assuming Gaussian distributions we get

$$
d \varphi=\sqrt{\Theta_{\text {Div }}^{2}+\Theta_{\text {Det }}^{2}+\Theta_{\text {Rough }}^{2}+\Theta_{\text {Refl }}^{2}+\Theta_{\text {Refr }}^{2}} .
$$

The transmitted intensity through the prisms is attenuated by absorption in the material and scattering at the surfaces.

Following these considerations we designed an energy analyser made up of prisms. For this purpose we used the Virtual Instrumentation Tool for the European Spallation Source (VITESS) [8] for which a new module was written. It simulates the refraction of neutrons by a given number of prisms and takes into account all of the above mentioned effects except for the surface scattering.

\section{Choice of the material}

We tested at the V14 neutron reflectometer at the HZB the applicability of $\mathrm{MgF}_{2}$ and silicon as refraction materials, because they are available as single crystals at reasonable prices and they show large ratios of refraction to absorption of 5.25 for $\mathrm{MgF}_{2}$ and 0.86 for Si. Considering the scattering on phonons [6] [7] these ratios decrease to 1.62 for $\mathrm{MgF}_{2}$ and 0.63 for silicon. The $\mathrm{MgF}_{2}$ prism row consists of a $50 \times 20 \times 2 \mathrm{~mm}^{3}$ single crystal block. The upper $0.5 \mathrm{~mm}$ of the material are mechanically cut into the shape of 33 prisms, each with an angle of $45 \mathrm{deg}$ to the base of the prisms.

The silicon prism rows are produced by anisotropic etching. They consist of 92 single prisms and cover an area of $65 \times 50 \mathrm{~mm}^{2}$. The height of each prism is $0.5 \mathrm{~mm}$. Since this is also the thickness of the raw material, there is no unnecessary material in the beam path. We used (100) oriented silicon wafers coated with silicon nitride. The prism structure was transferred by an UV lithographic process to the silicon nitride layer. During the etch process prisms with an angle of $54.7 \mathrm{deg}$ to the basis were emerging. 
We performed the measurements of refraction and transmission with a neutron beam of the wavelength $4.9 \AA$, a height of $0.3 \mathrm{~mm}$ and a divergence of $0.006 \mathrm{deg}$. To detect the refracted beam a $3 \mathrm{He}$ detector with a slit of $0.2 \mathrm{~mm}$ at $2 \mathrm{~m}$ behind the prisms was moved across the beam. For the $\mathrm{MgF}_{2}$ prism row we found the maximum intensity of the refracted beam at $0.08 \mathrm{deg}$, see Fig.1, which corresponds to a refraction of $0.0024 \mathrm{deg}$ per prism. This experimental result is in good agreement with the theoretical calculation. The discrepancy of $8 \%$ is due to mechanical imperfections of the prisms. The beam width increases while passing through the prisms by $0.015 \mathrm{deg}$ compared to the FWHM of the direct beam.

For the $92 \mathrm{Si}$ prisms we found the maximum intensity at $0.058 \mathrm{deg}$ (see Fig. 2 ), which corresponds to a refraction of 0.0006 deg per prism. This result is as well in good agreement with the calculation. The discrepancy of $5 \%$ is also caused by imperfections of the prisms. The beam width increases by $0.005 \mathrm{deg}$ compared to the FWHM of the direct beam.

To measure the transmission through the prism arrays the distance between sample and detector was reduced to $50 \mathrm{~cm}$ and the detector slit was removed. This increased the detector acceptance up to 2 deg which allows to cover the full refracted beam including the spread measured before. Through the 33 $\mathrm{MgF}_{2}$ prisms $86 \%$ of all incoming neutrons were transmitted. This leads to an attenuation coefficient of $\mu=0.055 \mathrm{~cm}^{-1}$, which is about $83 \%$ higher than the literature value including the phonon scattering [6]. The increase of the beam width and the high intensity losses indicate that the prisms surfaces are very rough and the neutrons are diffusely scattered.

The same measurement with $92 \mathrm{Si}$ prisms showed that $88 \%$ of the neutrons are transmitted. The attenuation coefficient is $\mu=0.03 \mathrm{~cm}^{-1}$, which differs $6 \%$ from the literature value.

Since the widening of the refracted beam and its attenuation is much smaller for the $\mathrm{Si}$ than for the $\mathrm{MgF}_{2}$ prisms, we decided to build and test a prism energy analyser consisting of $\mathrm{Si}$.

\section{Measurement of the refraction and resolution}

We performed the measurement of the energy analyser at the time-offlight reflectometer EROS at LLB. A stack of $4 \mathrm{Si}$ prism rows, each with a height of $0.25 \mathrm{~mm}$ and made up from 191 single prisms, was put $2 \mathrm{~m}$ in front of a single ${ }^{3} \mathrm{He}$ detector. The slit of the detector was set to $0.5 \mathrm{~mm}$. The detector and the slit were moved in steps of $0.5 \mathrm{~mm}$ in the direction of the 
refracted beam. The slit in front of the prisms was set to $0.3 \mathrm{~mm}$ height and the first slit $1800 \mathrm{~mm}$ upstream to $0.5 \mathrm{~mm}$, so the beam divergence of the incoming beam was limited to $0.013 \mathrm{deg}$.

The measurement in the time-of-flight mode gives as a result the refracted intensity of the different wavelengths at certain detector positions. Putting the plots for all detector positions together, we get a two dimensional map, which shows the intensity distribution according to the detector positions and the neutron wavelengths, see Fig. 3. The coarse structure is an artifact due to the width of the slit and the step width of the detector. Below the main intensity of the refracted beam there is an area with low intensity, where neutrons are detected due to the total reflection and small angle refraction of the neutrons described above.

The measured wavelength distribution at each detector position has been fitted with an Gaussian function to extract the central wavelength and the FWHM. Fig. 4 shows the relation between wavelength and the detector angle. The data are in very good agreement with the data from a VITESS simulation.

Assuming Gaussian distributions the FWHM of the $1 \%$ wavelength resolution of the chopper had been subtracted from the fitted FWHM of the measured wavelength distribution. Thus we determined the wavelength resolution of the energy analyser to be $5 \%$ for $6.7 \AA$ neutrons, see Fig. 5 .

To quantify the effect of the diffuse scattering a fit with equation 4 and $\mathrm{d} \varphi$ as the only free parameter was made, resulting in a value of $0.027 \mathrm{deg}$ for $\mathrm{d} \varphi$.

By a VITESS simulation it was possible to give values for all terms in equation 5. The value for $\Theta_{\text {Refr }}$ is zero, because in the case of 191 prisms and for neutrons of wavelength smaller than $10 \AA$ all refracted angles are smaller than the critical angle of $\mathrm{Si}$, the effect of small angle refraction does not occur.

Table 1 shows that the only cause of broadening which was not known or simulated, amounts to $(0.011 \pm 0.006)$ deg. This part is caused by the scattering at the prisms surface and imperfections in the prisms structure. Its uncertainty is due to the uncertainty of the measured wavelength resolution. Fig. 6 shows that the experimental transmission of the neutrons varies between $90 \%$ at $2.5 \AA$ and $10 \%$ at $8 \AA$. A VITESS simulation shows that these losses of intensity are dominated by the total reflection of the neutrons at the back side of the prisms. With the prisms height of $0.25 \mathrm{~mm}$ all neutrons with wavelengths above $10 \AA$ hit the back of the prisms in the layer above so 


\begin{tabular}{|c|c|}
\hline Term & Value [deg] \\
\hline \hline$\Theta_{D i v}$ & 0.013 \\
$\Theta_{D e t}$ & 0.011 \\
$\Theta_{\text {Refr }}$ & 0.000 \\
$\Theta_{\text {Refl }}$ & 0.018 \\
\hline
\end{tabular}

Table 1: Contribution of the different effects to the angular broadening of the beam.

the intensity of the beam transmitted within one prism layer reduces to zero, since the totally reflected neutrons leave the prism rows at wrong angles. This is a problem, because when a white beam is used only the projection of the measured data (see Fig. 3) to the ordinate is available. To prevent to detect neutrons at wrong angles it is possible to coat the prisms back side with an absorbing layer. This does not reduce the intensity of the useful neutrons and cleans up the signal in the detector.

With a bigger stack of prism rows it is possible to refract a larger white beam without additional intensity losses due to the absorption in the material. In comparison to the flat $\mathrm{MgF}_{2}$ prism presented by Cubitt [4] this is an advantage but we would need 550 prisms at a wavelength of $3.7 \AA$ and 340 prisms at $11 \AA$ to achieve the same resolution. This increase of the total number of prisms would reduce the intensity of the useful beam to zero due to the total reflection. A solution could be the bending of the prisms optimized for the transmission of a selected wavelength band. This way the distribution of the transmitted neutrons can be shifted to larger wavelengths and its intensity increased.

\section{Conclusion}

It could be shown that due to the better surface quality of the prisms $\mathrm{Si}$ is better suited as prism material than $\mathrm{MgF}_{2}$.

The energy distribution of a neutron beam could be analyzed by a stack of Si prism rows. We could achieve a resolution of better than $5 \%$ for neutrons with wavelengths longer than $6.7 \AA$.

The transmission varies between $84 \%$ for $2.5 \AA$ and $9 \%$ for $8 \AA$. These losses are dominated by internal reflections of the neutrons, which additionally lead to increased background. This problem can be overcome by coating the back side of the prisms with an absorbing layer and by bending the prism rows. The contribution of the surface roughness to the broadening of the angular 
range could be determined to be $(0.011 \pm 0.006)$ deg.

\section{Acknowledgements}

This research project has been supported by the European Commission under the 7th Framework Programme through "Research Infrastructures" action of the "Capacities" Programme, contract number CP-CSA_INFRA2008-1.1.1. Number 226507-NMI3.

\section{References}

[1] F. Ott, A. Menelle, Eur. Phys. J. Scecial Topics 167, 93 (2009)

[2] F. Ott, A. de Vismes, Physica B 397, 153 (2007)

[3] R. Cubitt, et al., Nucl. Instrum. Meth. A 558, 547 (2006)

[4] R. Cubitt, et al., Eur. Phys. L Plus 126, 111 (2011)

[5] W. Jark et al., J. Synchrotron Rad.11, 248-253 (2010)

[6] J. G. Barker, et al., Journal of Applied Crystallogrophy 41, 1003 (2008)

[7] A. W. Freund, Nucl. Instrum. Methods, 213, 495-501 (1983)

[8] VITESS web site: http://www.helmholtzberlin.de/forschung/grossgeraete/neutronenstreuung/projekte/vitess/index_de.html HZB (2012) 


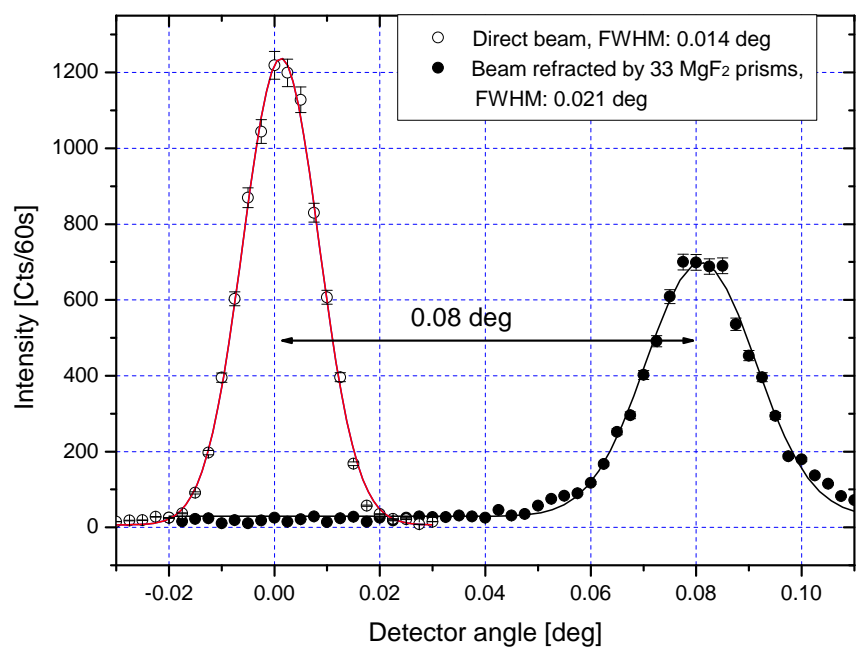

Figure 1: Refraction of a neutron beam with the wavelength $4.9 \AA$ by $33 \mathrm{MgF}_{2}$ prisms in comparison with the direct beam.

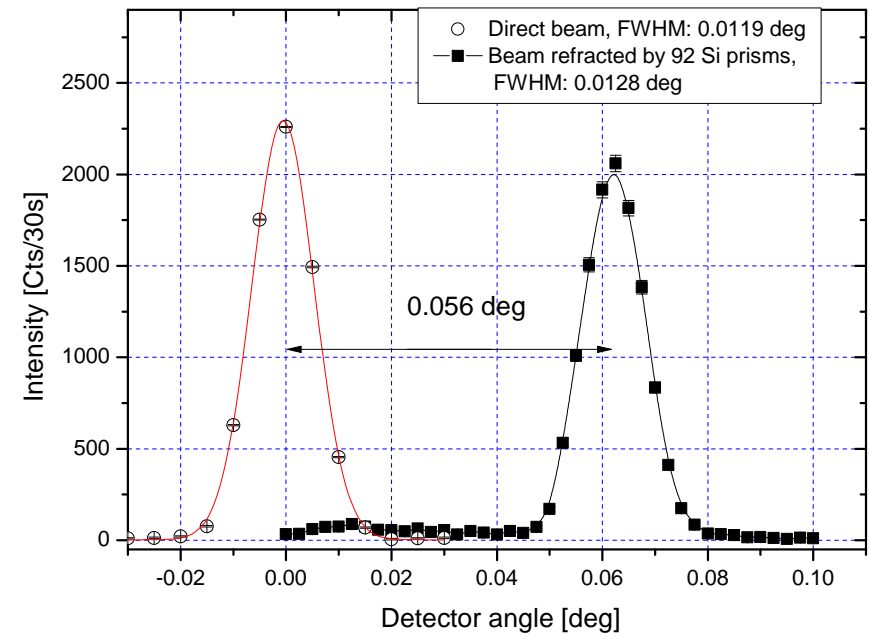

Figure 2: Refraction of a neutron beam with the wavelength $4.9 \AA$ by $92 \mathrm{Si}$ prisms in comparison with the direct beam. 


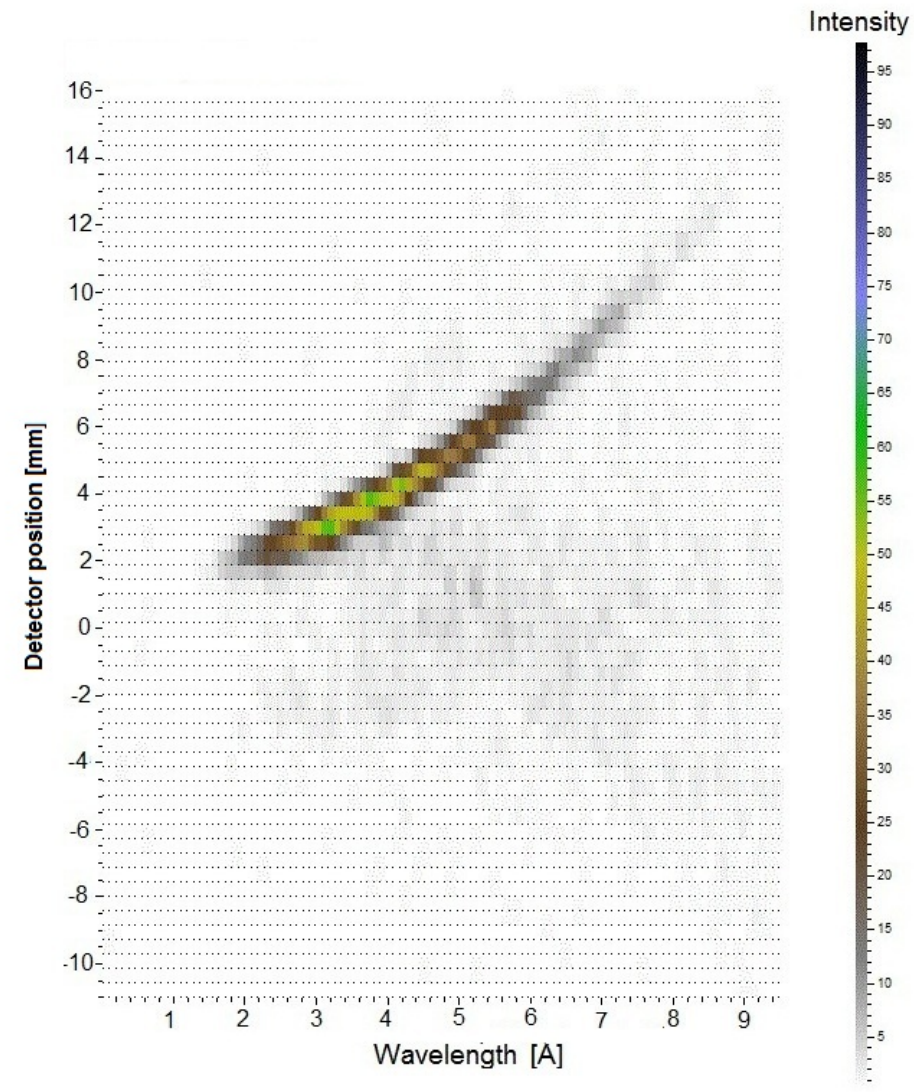

Figure 3: Measured intensity of the neutrons as a function of the neutron wavelength and the detector position after refraction by $191 \mathrm{Si}$ prisms. Due to the energy analyser each detector position encodes via the refracted angle the wavelength of the neutrons. 


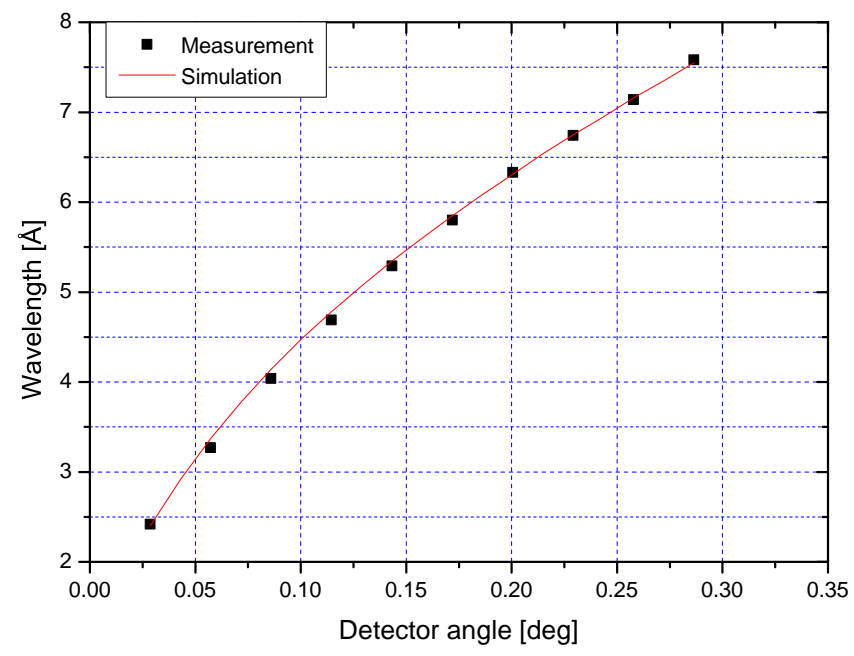

Figure 4: Measured main wavelength for each detector angle and the data from a VITESS simulation.

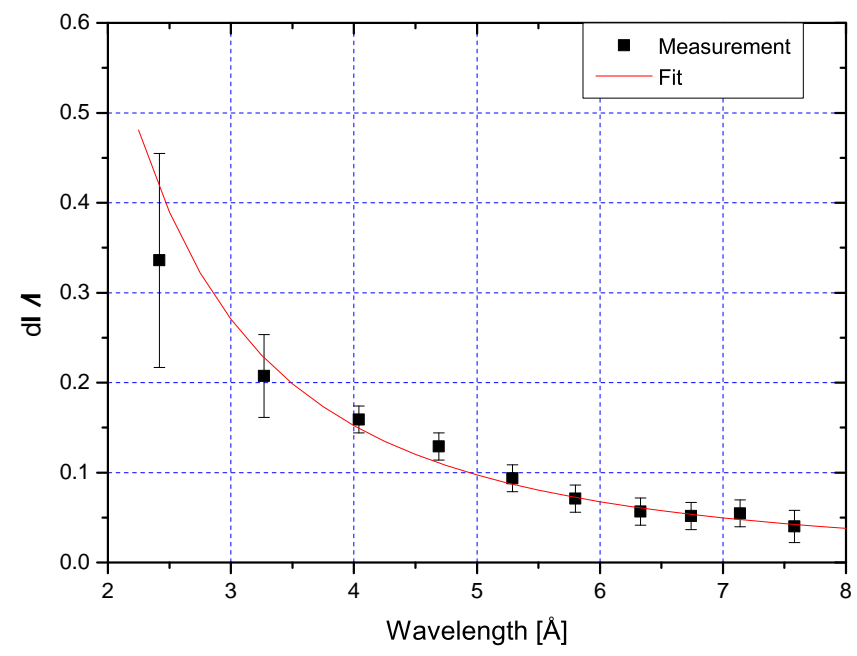

Figure 5: Experimental wavelength resolution of the energy analyser fitted with equation 4 and 5 . The free parameter $d \varphi$ was fitted to be $0.027 \mathrm{deg}$. 


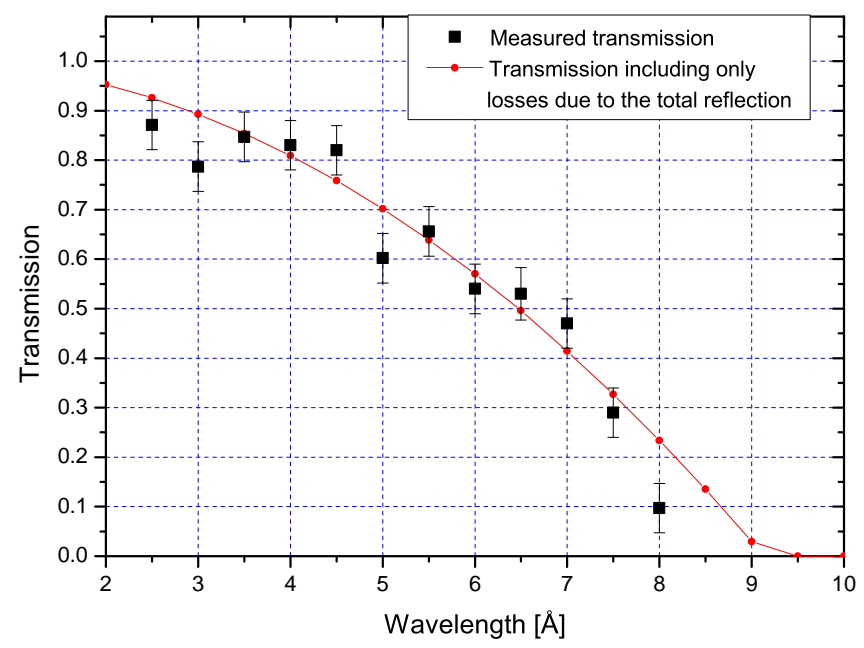

Figure 6: Measured transmission and calculated losses caused only by total reflection for 191 prisms. 